journal club

\title{
MR-gesteuerte Ultraschall-Läsion als neue Option
}

Fragestellung: Zur Behandlung schwerster Formen des essenziellen Tremors steht derzeit die tiefe Hirnstimulation als offenes operatives Verfahren und die Gamma-knife-Surgery [1] mit hochenergetischer Strahlung zur Verfügung. Die neu entwickelte MR-gesteuerte Ultraschall-Läsion (MRgUSL) kann hier möglicherweise ähnliche Resultate erzielen.

Hintergrund: Die Behandlung mit hochenergetischem und fokussiertem Ultraschall wird derzeit bei der Tumorbehandlung von peripheren Tumoren und Hirntumoren geprüft. Dabei werden hohe Energien fokal im Gewebe erzeugt, die zu einer Gewebeerwärmung und dadurch zu einer Läsion führen. Damit entsteht die Möglichkeit auch funktionelle Eingriffe unter anderem zur Besserung von Bewegungsstörungen zu entwickeln. Dieses wird hier in einer Proof-of-concept Studie beim essenziellen Tremor geprüft. Das an-

Lipsman N, Schwartz ML, Huang $\mathrm{Y}$ et al. MR-guided focused ultrasound thalamotomy for essential tremor: a proof-of-concept study. Lancet Neurology 2013; 12: 462-68 gezielte Areal ist der Vim des Thalamus. Die darunter liegende zona incerta ist das klassische Zielgebiet der früheren läsionellen Chirurgie und der heutigen tiefen Hirnstimulation.

\section{-Kommentar von Günther Deuschl, Kiel}

\section{Spannende Methode mit Potenzial}

Diese Proof-of-concept-Studie beweist fast nichts, dennoch ist der Inhalt des Papers wirklich wissenswert. Es wurde bei vier Patienten gezeigt, dass man mit Ultraschall ausreichend Energie im ZNS applizieren kann, um eine Thermokoagulation zu erreichen. Hier wird eine völlig neue Behandlungsmethode für Neurologie und Psychiatrie vorgestellt, deren Zukunft noch ungewiss ist [2]. Sie hat aber Potenzial, wenn sie in Zukunft ihre Sicherheit beweisen kann. Das Zielverfahren setzt exzellente Technologie voraus. Es scheint aber anwendbar, da es in den USA bereits für die Behandlung von Uterusfibromen zugelassen ist. Es ist noch unklar, ob das Testverfahren für die nachfolgende Läsion präzise genug ist und damit der endgültige Läsionsort genau genug festgelegt werden kann. Eine große Frage ist, ob bei intrakranieller Anwendung das Läsionsvolumen beim Eingriff ausreichend gut kontrolliert werden kann. Selbst wenn das der Fall ist, kennt man von der Gamma-knifeSurgery sich später ausdehnende Läsionen („running lesions“). Es muss gezeigt werden, dass diese bei der MRgUSL nicht vorkommen. Sollte sich das Verfahren in weiteren Pilotstudien bewähren, sind kontrollierte Langzeitstudien dringend erforderlich. In Zukunft wird das Verfahren vielleicht auch zur Pallidotomie für Morbus Parkinson und Dystonie erwogen. Nuc-
Methodik: Mit einem stereotaktisch plazierten Gerät mit 1.024 fokussierbaren Ultraschallköpfen wird der Vim beschallt. Dabei kann der Läsionsort, die Läsionsgröße und die Temperatur im Zielgebiet kontinuierlich mit der Kernspintomografie überprüft werden, da auch die subläsionelle Temperaturerhöhung mit dem MRI gemessen werden kann. Der Eingriff erfolgt im Wachzustand durch die intakte Kopfoberfläche. Vier Patienten wurden behandelt und die Therapieergebnisse mit Standardskalen erfasst.

Ergebnisse: Durch die Behandlung mit MRgUSL trat eine sofortige Besserung des Tremors ein mit einer mittleren Besserung um $89 \%$ nach einem Monat und $81 \%$ nach drei Monaten. Funktionelle Skalen verbesserten sich ebenfalls. An Nebenwirkungen wurden Parästhesien bei einem Patienten und eine tiefe Venenthrombose berichtet. Kernspintomografische Kontrolluntersuchungen zeigen innerhalb von drei Monaten keine ungewollte Ausdehnung der Läsion.

Schlussfolgerungen: Bei vier Patienten hatte das Verfahren keine ernsthaften Nebenwirkungen. Über drei Monate war bei diesen ein positiver Effekt zu verzeichnen. Prospektive Studien sind erforderlich.

leus-subthalamicus-Läsionen müssen extrem präzise lokalisiert werden. Deshalb und weil bei der Subthalamicus-Läsion schwere Dyskinesien auftreten können, erscheint diese Lokalisation wenig geeignet. Ein wahrscheinlich sehr großer Nachteil der Methode ist die Tatsache, dass das es sich um eine definitive Läsion handelt, die nicht mehr nachträglich angepasst werden kann. Dies ist die große Stärke der tiefen Hirnstimulation, die durch die MRgUSL nicht erreicht wird. Sollte aber eine Kontraindikation für die tiefe Hirnstimulation bestehen, so mag dieses Verfahren nützlich werden. Letztlich kehrt das Verfahren zur alten läsionellen Stereotaxie zurück, indem Thermoläsionen mit Ultraschallenergie erzeugt werden - allerdings ohne den Schädel zu eröffnen. Es sei angemerkt, dass heute bereits experimentell daran gearbeitet mit Ultraschall auch reversible neuronale Blockaden erzeugen zu können [3]. Dies könnte zur Entwicklung nicht invasiver Behandlungsmethoden mit Ultraschall führen.

\footnotetext{
Referenzen

1. Lim SY et al. Arch Neurol. 2010; 67: 584-8

2. Deuschl G. Lancet Neurology 2013; 12: $420-2$

3. Tufail $Y$ et al. Neuron 2010; 66: 681-94
} 